\title{
ON THE SPECTRA OF SEMI-NORMAL OPERATORS
}

\author{
BY \\ C. R. PUTNAM(1)
}

1. Introduction. Let $\mathfrak{H}$ denote a Hilbert space of elements $f, g, \cdots$, with the norm $\|f\|=(f, f)^{1 / 2}$. There will be considered only bounded operators, that is, linear transformations $T$ defined on the whole of $\mathfrak{H}$ and satisfying $\|T\|=\sup \|T f\|<\infty$, where $\|f\|=1$. The spectrum of $T$ will be denoted by $\operatorname{sp}(T)$, while the closure of the value domain of $T$, that is, the closure of the set of complex numbers $(T f, f)$ where $\|f\|=1$, will be denoted by $W(T)$. It is known (Hausdorff-Toeplitz; cf. Stone $[12$, p. 131]) that $W(T)$ is a closed convex set and always contains $\operatorname{sp}(T)$. An operator $T$ will be called semi-normal if

$$
T T^{*}-T^{*} T \equiv D \geqq 0 \text { or } D \leqq 0 .
$$

If $T$ is semi-normal with the Cartesian representation

$$
T=H+i J, \text { where } H=\left(T+T^{*}\right) / 2 \text { and } J=\left(T-T^{*}\right) / 2 i,
$$

then (1.1) holds if and only if

$$
H J-J H=i C, \quad \text { where } C \geqq 0 \text { or } C \leqq 0 \text { (with } D=2 C \text { ). }
$$

In particular $T$ is normal if $C$ (or $D$ ) is 0 .

By an isolated part $\sigma$ of $\operatorname{sp}(T)$ is meant a subset of $\operatorname{sp}(T)$ which lies at a positive distance from its complementary part $\operatorname{sp}(T)-\sigma$; see Riesz and Sz-Nagy [10, pp. $418 \mathrm{ff}$.]. It is known that if $\sigma$ is an isolated part of $\operatorname{sp}(T)$, then there exists a "parallel projection" $P=P_{\sigma}$, a bounded operator, not necessarily self-adjoint, satisfying $P^{2}=P$ and such that both $P \mathfrak{G}$ and $(I-P) \mathfrak{H}$ are invariant under $T$. Moreover $\operatorname{sp}\left(T^{\prime}\right)=\sigma$, where $T^{\prime}=T / P \mathfrak{H}$ denotes the restriction of $T$ to the space $P \mathfrak{G}$. In case $T$ is semi-normal, so also is $T^{\prime}$; cf. Berberian $[1$, p. 161 , problem 10].

In case $A$ is a self-adjoint operator with the spectral resolution

$$
A=\int \lambda d E(\lambda)
$$

then the set $\mathfrak{H}_{a}$ of elements $f$ in $\mathfrak{H}$ for which $\|E(\lambda) f\|^{2}$ is an absolutely continuous function of $\lambda$ is known to be a subspace of $\mathfrak{S}$; see Halmos [2, p. 104].

Received by the editors September 23, 1963 and, in revised form, January 3, 1964.

(1) This work was supported by the National Science Foundation research grants NSFG18915, GP-1665. 
Ordinary one and two dimensional Lebesgue measure of a corresponding Borel set $S$ of the line or plane will be denoted respectively by $\mu_{1}(S)$ and $\mu_{2}(S)$. If $S$ is a Borel set of the real line then the spectral family $\{E(\lambda)\}$ of (1.4) assigns a (self-adjoint) projection measure $E(S)$; see Halmos [2, pp. $58 \mathrm{ff}]$.

In $\S 2$ there will be stated several results for semi-normal operators $T$ which represent generalizations of corresponding results for normal operators. $\$ 3$ is concerned with estimates for $\|D\|$ (see (1.1)) involving the areas of the sets $W(T)$ and $\operatorname{sp}(T)$. Further results on the nature of the spectrum of $T$ are given in $\S \S 4$ and 5. Some remarks on absolute continuity of the real and imaginary parts of $T$ are made in $\S 6 . ~ \S \S 7-13$ contain the proofs of the theorems. The last two $\S \S 14$ and 15 are devoted to a few applications of the results to Toeplitz matrices and singular integral operators.

2. THEOREM I. Let $T$ of (1.2) be semi-normal, so that (1.1) or (1.3) holds (i) If $x_{0} \in \mathrm{sp}(H)$ there exists some real number $y_{0}^{\prime}$ and a sequence $\left\{h_{n}\right\}$ of unit vectors for which $\left(H-x_{0} I\right) h_{n} \rightarrow 0$ and $\left(J-y_{0}^{\prime} I\right) h_{n} \rightarrow 0$ as $n \rightarrow \infty$ so that, in particular, $x_{0}+i y_{0}^{\prime} \in \mathrm{sp}(T)$. Similarly, if $y_{0} \in \operatorname{sp}(J)$ there exists some real number $x_{0}^{\prime}$ and a sequence $\left\{j_{n}\right\}$ of unit vectors for which $\left(H-x_{0}^{\prime} I\right) j_{n} \rightarrow 0$ and $\left(J-y_{0} I\right) j_{n} \rightarrow 0$ as $n \rightarrow \infty$ so that, in particular, $x_{0}^{\prime}+i y_{0} \in \operatorname{sp}(T)$. (ii) If $x_{0}$ and $y_{0}$ are real and if $x_{0}+i y_{0} \in \operatorname{sp}(T)$ then $x_{0} \in \operatorname{sp}(H)$ and $y_{0} \in \operatorname{sp}(J)$.

It follows from the above theorem that the spectra of the real and imaginary parts respectively of a semi-normal operator are precisely the sets of real numbers obtained by projecting the spectrum of $T$ onto the $x$ - and $y$-axes. This result for normal operators is known and can be deduced, for instance, from the spectral resolution formula.

There follows immediately the

Corollary 1 OF THeOREM I. If $T$ is semi-normal and if $\operatorname{sp}(T)$ is real then $T$ is self-adjoint.

Another consequence is

Corollary 2 OF THEOREM I. If $T$ is semi-normal then the set $W(T)$ is the smallest closed convex set containing the spectrum of $T$.

In order to prove Corollary 2 , note that for a self-adjoint operator $A$, the set $W(A)$ is always the closed segment of the real axis joining the maximum and minimum points of $\operatorname{sp}(A)$. In addition, if $\theta$ is real, then

$$
T_{\theta}=T e^{i \theta}
$$

is also semi-normal. Since $\operatorname{sp}\left(T_{\theta}\right)=e^{i \theta} \operatorname{sp}(T)$ and $W\left(T_{\theta}\right)=e^{i \theta} W(T)$, it follows from Theorem I that $W(T)$ is contained in every closed rectangle of the complex plane which contains $\operatorname{sp}(T)$. Thus $W(T)$ is contained in the intersection of all 
such rectangles, that is, $W(T)$ is contained in the least closed convex set containing $\operatorname{sp}(T)$. Since, even for arbitrary $T, \operatorname{sp}(T)$ is always a subset of $W(T)$, the proof of the corollary is complete.

In case $T$ is normal the assertion of Corollary 2 is known (Toeplitz).

3. Areas of $W(T)$ and $\operatorname{sp}(T)$. Let $T$ be arbitrary and define the function $M(x)$ on $-\infty<x<\infty$ by

$$
M(x)=\left\{\begin{array}{l}
\sup \operatorname{Im}(z)-\inf \operatorname{Im}(z), \text { where } z \in \operatorname{sp}(T) \text { and } x=\operatorname{Re}(z), \\
0 \text { if } x \notin \operatorname{Re}(\operatorname{sp}(T)) .
\end{array}\right.
$$

Thus, for $x \in \operatorname{Re}(\operatorname{sp}(T)), M(x)$ is the distance between the upper and lower boundaries of $\operatorname{sp}(T)$ over $x$. For every real $\theta$ define $T_{\theta}$ by (2.1) and let the function $M_{\theta}(x)$ correspond to $T_{\theta}$ as $M(x)\left(=M_{0}(x)\right)$ does to $T\left(=T_{0}\right)$.

THEOREM II. Let $T$ be semi-normal, so that (1.1) holds. Then for every real $\theta$,

$$
\pi\|D\| \leqq \int M_{\theta}(x) d x
$$

More generally, if $H_{\theta}=\operatorname{Re}\left(T_{\theta}\right)$ has the spectral resolution

$$
H_{\theta}=\int \lambda d E_{\theta}(\lambda)
$$

and if $S$ denotes any Borel set of the real axis, then

$$
\pi\left\|E_{\theta}(S) D E_{\theta}(S)\right\| \leqq \int_{S} M_{\theta}(x) d x .
$$

That, in fact, relation (3.4) implies (3.2) follows from the observation that if $S=(-\infty, \infty)$ then $E_{\theta}(S)=I$.

In case $S$ is a Borel set of measure 0, relation (3.4) implies that $E_{\theta}(S) D E_{\theta}(S)=0$. Since $D$ is semi-definite, then $D E_{\theta}(S)=0$, a result proved in [5]. See also the remarks of $\S 6$ below.

In order to clarify the assertion of Theorem II a few consequences will be noted. First there follows the

COROllary 1 OF TheOREM II. If $T$ satisfies (1.1) then

$$
\pi\|D\| \leqq \mu_{2}(W(T)) \text {. }
$$

In order to prove (3.5) let $\theta$ be fixed. It is clear from the definition of $M_{\theta}(x)$ and the fact that $\operatorname{sp}(T)$ is contained in $W(T)$ that, for $x \in \operatorname{Re}\left(\operatorname{sp}\left(T_{\theta}\right)\right), M_{\theta}(x)$ is not greater than the distance between those points of the upper and lower boundaries of the set $W\left(T_{\theta}\right)$ which lie over the point $x$ of the real axis. Thus the right side of (3.2) is not greater than the area of $W(T)$, and (3.5) follows.

It can be noted that the above corollary implies Corollary 1 of Theorem I. 
Corollary 2 OF TheOREM II. Let $T$ satisfy (1.1). Suppose that for some fixed $\theta$ the set $e^{i \theta} \operatorname{sp}(T)$ has the property that, except possibly for a set of real values $x$ of measure 0 , the set $S_{x}=\left\{z: z \in e^{i \theta} \operatorname{sp}(T)\right.$ and $\left.\operatorname{Re}(z)=x\right\}$ is either a closed interval, or a single point, or the empty set. Then

$$
\pi\|D\| \leqq \mu_{2}(\operatorname{sp}(T)) .
$$

The proof follows from (3.2) if it is noted that $\mu_{2}\left(e^{i \theta} \operatorname{sp}(T)\right)=\mu_{2}(\operatorname{sp}(T))$ and that, in the present case, for almost all $x, M_{\theta}(x)=\mu_{1}\left(S_{x}\right)$ for $x \in \operatorname{Re}\left(e^{i \theta} \operatorname{sp}(T)\right)$ and $M_{\theta}(x)=0$ otherwise.

The restriction imposed on $\operatorname{sp}(T)$ by the hypothesis of the preceding corollary is that there should exist some direction, determined by a line $L$, with the property that almost all sections of $\operatorname{sp}(T)$, obtained by intersections of $\operatorname{sp}(T)$ with lines parallel to $L$, should be intervals or points.

The inequalities (3.5) and (3.6) are optimal in the sense that there exist seminormal operators $T$ which are not normal and for which both (3.5) and (3.6) become equalities. In fact, if $T$ is isometric but not unitary, then $T^{*} T=I$ while $T T^{*}$ is singular. It is easily verified that $\|D\|=1$. Also both $\operatorname{sp}(T)$ and $W(T)$ are the closed unit disk $|z| \leqq 1$ (see, e.g., [6, p. 1650]) and so equality holds in (3.5) and (3.6).

Whether (3.6) must hold for all semi-normal operators will remain undecided. In fact, the question will remain open as to whether

$$
\mu_{2}(\operatorname{sp}(T))>0
$$

holds for all semi-normal, but not normal, operators. That (3.7) is satisfied in certain special cases however was shown by Putnam [6], Stampfli [11].

4. Isolated parts of $\operatorname{sp}(T)$. Let $T$ satisfy (1.1) and let

$$
\Omega=\text { smallest subspace of } \mathfrak{S} \text { reducing } T \text { and containing } \Re_{D},
$$

where $\Re_{D}$ denotes the range of $D$. Thus, the orthogonal complement $\Omega^{\perp}$ of $\Omega$ is the largest subspace of $\mathfrak{H}$ reducing $T$ and contained in the null space of $D$; or, equivalently, $\Omega^{\perp}$ is the largest subspace reducing $T$ on which $T$ is normal. It will be supposed that $T$ is not normal on $\mathfrak{H}$, so that $\Omega \neq 0$. The assertion of the next theorem will relate to the operator $T$ on $\Omega$ and it can therefore be supposed that $\Omega=\mathfrak{H}$.

THEOREM III. Consider the semi-normal operator $T$ as an operator on the space $\mathfrak{H}=\Omega(\neq 0)$, so that there do not exist any nontrivial subspaces reducing $T$ on which $T$ is normal. For each real $\theta$, let $T_{\theta}$ be defined by (2.1).

(i) If $S$ is a Borel set on the real axis, then

$$
M_{\theta}(x)=0 \text { a.e. on } S \text { implies } E_{\theta}(S)=0,
$$

where $E_{\theta}(\lambda)$ is defined by (3.3). 
(ii) Let $\sigma$ be any isolated part of $\operatorname{sp}(T)$ with the parallel projection $P$ (see $\S 1$ ) and let $T^{\prime}=T / P \mathfrak{W}$ denote the restriction of $T$ to the subspace $P \mathfrak{W}$. Let $H_{\theta}^{\prime}=\operatorname{Re}\left(T_{\theta}\right)$, where $T_{\theta}^{\prime}=T^{\prime} e^{i \theta}$, and suppose that $H_{\theta}^{\prime}$ has the spectral resolution

$$
H_{\theta}^{\prime}=\int \lambda d E_{\theta}^{\prime}(\lambda)
$$

Then

$$
M_{\theta}^{\prime}(x)=0 \text { a.e. on } S \text { implies } E_{\theta}^{\prime}(S)=0,
$$

where $M_{\theta}^{\prime}(x)$ corresponds to $T^{\prime}$ as $M_{\theta}(x)$ does to $T$.

The above theorem has various implications concerning the nature of the spectrum of a semi-normal operator $T$. Since a normal operator is also seminormal and since any closed bounded set is the spectrum of some normal operator, it is clear that the investigation of $\operatorname{sp}(T)$ when $T$ is semi-normal should be restricted to the case $\mathfrak{S}=\boldsymbol{\Omega}$ as in Theorem III.

Corollary I OF TheOREM III. Let $T$ be semi-normal, suppose $\mathfrak{S}=\Omega(\neq 0)$ as in Theorem III, and let $\sigma$ denote any isolated part of $\operatorname{sp}(T)$. Let $Q$ denote any open strip of the complex plane bounded by two parallel lines and such that the set $\sigma \cap Q$ is not empty. Then $\sigma \cap Q$ is not a subset of any set $N$ with the following property: for some $\theta$, the strip $Q e^{i \theta}$ is perpendicular to the $x$-axis, intersects the $x$-axis in an open interval $(\alpha, \beta)$, and the set $N e^{i \theta}$ is given by

$$
\left.N e^{i \theta}=\{x, f(x)): \alpha<x<\beta, f(x) \text { single-valued }\right\} .
$$

In fact, if the assertion were false, then $\sigma \cap Q$ would be a nonempty subset of some set $N$ of the type described. Since $\sigma=\operatorname{sp}\left(T^{\prime}\right)$ (cf. $\S 1$ ), then $\sigma e^{i \theta}$ is the spectrum of $T_{\theta}^{\prime}=T^{\prime} e^{i \theta}$, while

$$
\sigma e^{i \theta} \cap Q e^{i \theta} \text { is not empty }
$$

and

$$
\left(\rho e^{i \theta} \cap Q e^{i \theta}\right) \text { is a subset of } N e^{i \theta} .
$$

But (4.5) and (4.7) imply that $M_{\theta}^{\prime}(x)=0$ on $(\alpha, \beta)$ and so, by $(4.4), E_{\theta}^{\prime}((\alpha, \beta))=0$. According to Theorem I this implies that the set $\operatorname{Re}\left(\operatorname{sp}\left(T_{\theta}^{\prime}\right)\right) \cap(\alpha, \beta)$ is empty, in contradiction with (4.6). This proves the corollary.

It is seen that the above corollary implies that when $\mathfrak{G}=\Omega$, no isolated part of $\operatorname{sp}(T)$ is contained in a segment (cf. Corollary 1 of Theorem I) or, for instance, in a proper subset of the boundary of a rectangle or a circle. On the other hand, the possibility that an isolated part of $\operatorname{sp}(T)$ might consist of the entire boundary of a rectangle or circle is not ruled out. Actually, it will remain undecided whether such a situation is possible, or more generally, whether or not an isolated part of $\operatorname{sp}(T)$ (assuming $\mathfrak{T}=\Omega$ ) must have a positive two dimensional Lebesgue 
measure (cf. the end of $\$ 3$ ). However, there will be proved the following somewhat curious result.

\section{THEOREM IV. Let $T$ of (1.2) satisfy (1.1). Then either}

$$
\text { both } \operatorname{sp}(H) \text { and } \operatorname{sp}(J) \text { contain an interval, }
$$

or (3.6) holds.

Of course, if $T$ is normal, then $D=0$ and (3.6) certainly holds, while the assertion (5.1) may be false. On the other hand, if $D \neq 0$, not only the general validity of (3.6) but also that of (5.1) will remain undecided. However, there do exist estimates similar to (3.6) for the real and imaginary parts of $T$ and in which the two dimensional measure is replaced by one dimensional measure. In fact it was shown in [9] that whenever $T$ of (1.2) satisfies (1.1), then

$$
\pi\|D\| \leqq 2\|J\| \mu_{1}(\operatorname{sp}(H))
$$

and

$$
\pi\|D\| \leqq 2\|H\| \mu_{1}(\operatorname{sp}(J))
$$

A result similar to Theorem IV is

THEOREM V. Let $T$ of (1.2) satisfy (1.1) and suppose that $\mathfrak{H}=\Omega(\neq 0)$ where $\Omega$ is defined by (4.1), so that $T$ possesses no nontrivial reducing subspaces on which it is normal. If $\operatorname{sp}(T)$ has zero area, that is, if

$$
\mu_{2}(\operatorname{sp}(T))=0,
$$

then there exist two open sets whose closures are respectively the sets $\operatorname{sp}(H)$ and $\operatorname{sp}(J)$.

As noted above it is conceivable that the assertion of Theorem $\mathrm{V}$ is vacuous in the sense that the hypothesis (5.4) may never hold (when $D \neq 0$ ). Also, it will remain undecided whether the assertion of Theorem $\mathrm{V}$ always holds even without the assumption (5.4).

6. Remarks. Concerning the spectrum of semi-normal operators, it can be mentioned that the real and imaginary parts, $H_{\theta}$ and $J_{\theta}$, of $T_{\theta}$ are absolutely continuous on the space $\Omega$ defined by (4.1); [9]. In particular, if $Z$ is a Borel set of zero Lebesgue measure, necessarily $E_{\theta}(Z)=0$. In this connection, see (3.4) and (4.2).

7. Proof of (i) of Theorem I. Since $i T$ is also semi-normal and has the Cartesian form $i T=(-J)+i H$, it is clearly sufficient to prove only the first part of (i). It will be clear from the proof that there is no loss of generality in supposing that $D \geqq 0$. 
Let $x_{0} \in \operatorname{sp}(H)$. Then there exists a sequence $\left\{f_{n}\right\}$ satisfying

$$
\left(H-x_{0} I\right) f_{n} \rightarrow 0, \quad\left\|f_{n}\right\|=1,
$$

hence also,

$$
J\left(H-x_{0} I\right) f_{n} \rightarrow 0 .
$$

But, by (1.3),

$$
\left(H-x_{0} I\right) J-J\left(H-x_{0} I\right)=i C,
$$

and so, by (7.1), $\left(f_{n},\left(H-x_{0} I\right) J f_{n}\right)-\left(f_{n}, J\left(H-x_{0} I\right) f_{n}\right)=i\left\|C^{1 / 2} f_{n}\right\|^{2} \rightarrow 0$. Hence $C f_{n}=C^{1 / 2}\left(C^{1 / 2} f_{n}\right) \rightarrow 0$ and so, by (7.2) and (7.3), $\left(H-x_{0} I\right) J f_{n} \rightarrow 0$. Similarly, if $J f_{n}$ is now identified with the previous $f_{n}$, then $\left(H-x_{0} I\right) J^{2} f_{n} \rightarrow 0$ and, in like manner, $\left(H-x_{0} I\right) p(J) f_{n} \rightarrow 0$, where $p(J)$ denotes any polynomial in $J$. Hence if $\phi(\lambda)$ denotes any continuous function on $-\infty<\lambda<\infty$ and if $\phi(J)$ is defined by the usual functional calculus, then $\phi(J)$ can be approximated uniformly by polynomial operators $p(J)$ and so

$$
\left(H-x_{0} I\right) \phi(J) f_{n} \rightarrow 0, \quad\left\|f_{n}\right\|=1 .
$$

Next, let $J$ have the spectral resolution

$$
J=\int \lambda d F(\lambda)
$$

and suppose that $\operatorname{sp}(J)$ is contained in the interior of $\Delta_{1}=[c, d]$. Then $\left\|F\left(\Delta_{1}\right) f_{n}\right\|=1$ for all $n$. Clearly, for at least one of the intervals $\Delta=\left[c, \frac{1}{2}(c+d)\right]$ or $\Delta=\left[\frac{1}{2}(c+d), d\right]$, say $\Delta=\Delta_{2}$,

$$
\left\|F\left(\Delta_{2}\right) f_{n}^{(2)}\right\| \geqq 1 / 2 \quad(n=1,2, \cdots),
$$

where $\left\{f_{n}^{(2)}\right\}$ is a subsequence of $\left\{f_{n}^{(1)}\right\}$, with $f_{n}^{(1)}=f_{n}$. Continuing this process one obtains intervals $\Delta_{1}, \Delta_{2}, \cdots$ with the properties that for each fixed $k=1,2, \cdots$, $\Delta_{k+1}$ is contained in $\Delta_{k}$, the length of $\Delta_{k}$ is $(d-c) / 2^{k-1},\left\{f_{n}^{(k+1)}\right\}$ is a subsequence of $\left\{f_{n}^{(k)}\right\}$ and

$$
\left\|F\left(\Delta_{k}\right) f_{n}^{(k)}\right\| \geqq \frac{1}{2^{k-1}} \quad(k, n=1,2, \cdots) .
$$

Let $y_{0}^{\prime}$ denote the real number determined by the nested sequence of intervals $\left\{\Delta_{k}\right\}$, so that

$$
c_{k}, d_{k} \rightarrow y_{0}^{\prime} \text { as } k \rightarrow \infty \text {, where } \Delta_{k}=\left[c_{k}, d_{k}\right] \text {. }
$$

For each $k=1,2, \cdots$, choose $\gamma_{k}>0$ so that

$$
\gamma_{k} \rightarrow 0 \text { as } k \rightarrow \infty \text {, }
$$

and define the continuous function $\phi_{k}(\lambda)$ on $-\infty<\lambda<\infty$ as the function whose 
graph is the real axis from $-\infty$ to $\left(c_{k}-\gamma_{k}, 0\right)$, the three segments joining $\left(c_{k}-\gamma_{k}, 0\right)$ to $\left(c_{k}, 1\right)$ to $\left(d_{k}, 1\right)$ to $\left(d_{k}+\gamma_{k}, 0\right)$ and the real axis from $\left(d_{k}+\gamma_{k}, 0\right)$ to $\infty$.

Clearly,

$$
0 \leqq \frac{1}{2^{k-1}} \leqq\left\|F\left(\Delta_{k}\right) f_{n}^{(k)}\right\| \leqq\left\|\phi_{k}(J) f_{n}^{(k)}\right\| \leqq\left\|f_{n}^{(k)}\right\| .
$$

On putting $g_{k n}=\phi_{k}(J) f_{n}^{(k)} /\left\|\phi_{k}(J) f_{n}^{(k)}\right\|$, it is seen that $\left\|g_{k n}\right\|=1$ and, from (7.4) that for each fixed $k$,

$$
\left(H-x_{0} I\right) g_{k n} \rightarrow 0 \text { as } n \rightarrow \infty \text {. }
$$

On the other hand, it is clear from the definition of the $g_{k n}$ that

$$
\left\|\left(J-y_{0}^{\prime} I\right) g_{k n}\right\| \leqq d_{k}-c_{k}+\gamma_{k} .
$$

It now follows from (7.11) and (7.12), together with (7.8) and (7.9), that a subsequence $\left\{m_{k}\right\}$ of the positive integers can be chosen so that for $h_{k}=g_{k m_{k}}$, both $\left(H-x_{0} I\right) h_{k} \rightarrow 0$ and $\left(J-y_{0}^{\prime} I\right) h_{k} \rightarrow 0$ hold as $k \rightarrow \infty$, as was to be shown. This completes the proof of (i) of Theorem $I$.

8. Proof of (ii) of Theorem I. Let $q=x_{0}+i y_{0} \in \operatorname{sp}(T)$. It will be shown that $x_{0} \in \operatorname{sp}(H)$. (The argument that $y_{0} \in \operatorname{sp}(J)$ is similar.) Again it can be supposed without loss of generality that $D \geqq 0$. If $T_{z}=T-z I$, then it is seen from(1.2) that

$$
T_{z} T_{z}^{*}=(H-x I)^{2}+(J-y I)^{2}+C, \text { where } z=x+i y .
$$

In case $T_{q} T_{q}^{*}$ is singular, there exists a sequence $\left\{f_{n}\right\}$ of unit vectors satysfying $\left(T_{q} T_{q}^{*} f_{n}, f_{n}\right) \rightarrow 0$. Since $C \geqq 0$, this implies by (8.1) that $\left(H-x_{0} I\right) f_{n} \rightarrow 0$ and $\left(J-y_{0} I\right) f_{n} \rightarrow 0$, and (ii) is proved. In case $T_{q} T_{q}^{*}>0$ then necessarily $T_{q}^{*} T_{q}$ is singular and it follows from [6, p. 1650], that there exists a whole disk about $q$ lying in $\operatorname{sp}(T)$. Let then $y_{0}^{\prime}$ be defined as the maximum value $y$ with the property that, for $z=x_{0}+i y, T_{z}$ is singular. Clearly, $r=x_{0}+i y_{0}^{\prime} \in \operatorname{sp}(T)$ and $T_{r} T_{r}^{*}$ must be singular. Consequently, it follows as before that $x_{0} \in \operatorname{sp}(H)$ (as well as $\left.y_{0}^{\prime} \in \operatorname{sp}(J)\right)$. This completes the proof of (ii).

9. Proof of Theorem II. Let the real part $H$ of the semi-normal operator $T$ have the spectral resolution

$$
H=\int \lambda d E(\lambda)
$$

and let $\Delta=(a, b]$ denote a half-open interval of the $\lambda$-axis. For the projection $E(\Delta)=E(b)-E(a)$ and for an arbitrary operator $A$, put $A_{\Delta}=E(\Delta) A E(\Delta)$. Then $A_{\Delta}$ leaves invariant the Hilbert space $\mathfrak{H}_{\Delta}=E(\Delta) \mathfrak{H} ; \operatorname{sp}\left(A_{\Delta}\right)$ will denote the spectrum of $A_{\Delta}$ as an operator on $\mathfrak{S}_{\Delta}$.

It was shown in [9] that (1.1)-(1.3) imply (5.3), that is,

$$
\pi\|C\| \leqq\|H\| \mu_{1}(\operatorname{sp}(J)) .
$$


Now, it follows from (1.2) and (1.3) that $T_{\Delta}=H_{\Delta}+i J_{\Delta}$ and

$$
H_{\Delta} J_{\Delta}-J_{\Delta} H_{\Delta}=i C_{\Delta} \text {. }
$$

Since $C_{\Delta} \geqq 0$ or $C_{\Delta} \leqq 0$ according as $C \geqq 0$ or $C \leqq 0$, then $T_{\Delta}$ is semi-normal on $\mathfrak{H}_{\Delta}$. If $\mu$ is arbitrary, then (9.3) implies that

$$
\left(H_{\Delta}-\mu I_{\Delta}\right) J_{\Delta}-J_{\Delta}\left(H_{\Delta}-\mu I_{\Delta}\right)=i C_{\Delta} \text {. }
$$

Hence, if $\mu$ is chosen to be the midpoint of $\Delta$ and if $d$ denotes the length of $\Delta$, then $\left\|H_{\Delta}-\mu I_{\Delta}\right\| \leqq d / 2$, and it follows from a relation similar to (9.2) but in which $H$ and $J$ are replaced by $H_{\Delta}-\mu I_{\Delta}$ and $J_{\Delta}$, that

$$
(2 \pi)^{1 / 2}\left\|C^{1 / 2} E(\Delta) f\right\| \leqq\left[d \mu_{1}\left(\operatorname{sp}\left(J_{\Delta}\right)\right)\right]^{1 / 2}\|E(\Delta) f\| .
$$

Let $y_{0} \in \operatorname{sp}\left(J_{\Delta}\right)$. Then by Theorem $I$, there exists some $x_{0}$ and a sequence $\left\{f_{n}\right\}$ of unit vectors in $\mathfrak{S}_{\Delta}$, thus $\left\|f_{n}\right\|=1$ and $f_{n}=E(\Delta) f_{n}$, for which

$$
\left(H-x_{0} I\right) f_{n} \rightarrow 0
$$

and

$$
E(\Delta)\left(J-y_{0} I\right) f_{n} \rightarrow 0 .
$$

(Note that $E(\Delta) H E(\Delta)=H E(\Delta)$, also that $x_{0} \in \operatorname{sp}\left(H_{\Delta}\right)$ and hence $x_{0} \in \Delta^{*}$, the closure of $\Delta$.)

Now, relation (9.7) implies that

$$
\left(\left(J-y_{0} I\right) f_{n}, f_{n}\right) \rightarrow 0,
$$

from which it follows that there exists some pair of real numbers $y_{1}$ and $y_{2}$ satisfying

$$
y_{1} \leqq y_{0} \leqq y_{2} \text { and } y_{1}, y_{2} \in \operatorname{sp}(J) .
$$

(The possibility $y_{1}=y_{2}$ is allowed.)

Next, it will be shown that there exists a point $y_{1}^{\prime} \leqq y_{0}$ for which $x_{0}+i y_{1}^{\prime} \in \operatorname{sp}(T)$. To this end, note that if $\left(J-y_{0} I\right) f_{n} \rightarrow 0$ as $n \rightarrow \infty$, then in fact $y_{0}^{\prime}$ can be chosen to be $y_{0}$. Consequently it can be supposed that

$$
\lim \sup \left\|\left(J-y_{0} I\right) f_{n}\right\|>0, \quad n \rightarrow \infty .
$$

As in $\S 7$, suppose that $\operatorname{sp}(J)$ is contained in the interior of $[c, d]$ so that by (9.9), $c<y_{0}$. If $\Delta_{1}=\left[c, y_{0}\right]$, it follows from (9.8) and (9.10) that

$$
\lim \sup \left\|F\left(\Delta_{1}\right)\left(J-y_{0} I\right) f_{n}\right\|>0, \quad n \rightarrow \infty,
$$

where $F(\lambda)$ is defined by (7.5). (In fact, if (9.11) were false, it would follow from (9.8) that $\int\left|\lambda-y_{0}\right| d\left\|F f_{n}\right\|^{2} \rightarrow 0$ and hence $\int\left(\lambda-y_{0}\right)^{2} d\left\|F f_{n}\right\|^{2} \rightarrow 0$, in contradiction with (9.10).) Hence there exists a subsequence $\left\{g_{n}\right\}$ of $\left\{f_{n}\right\}$ for which 


$$
\left(H-x_{0} I\right) g_{n} \rightarrow 0
$$

and

$$
\left\|F\left(\Delta_{1}\right) g_{n}\right\|>\text { const. }>0 \text {. }
$$

The argument of $\S 7$ can now be applied so as to yield a point $y_{1}^{\prime}$ belonging to $\Delta_{1}$, hence $y_{1}^{\prime} \leqq y_{0}$, and a sequence $\left\{h_{n}\right\}$ of unit vectors for which

$$
\left(H-x_{0} I\right) h_{n} \rightarrow 0 \text { and }\left(J-y_{1}^{\prime} I\right) h_{n} \rightarrow 0, \quad n \rightarrow \infty .
$$

(Note that the present $\Delta_{1}$ plays the role of $[c, d]$ in the argument of $\left.\S 7.\right)$ Thus, $z_{1}=x_{0}+i y_{1}^{\prime} \in \operatorname{sp}(T) ;$ a similar argument shows that $z_{2}=x_{0}+i y_{2}^{\prime} \in \operatorname{sp}(T)$ for some $y_{2}^{\prime} \geqq y_{0}$.

Consequently, whenever $y_{0} \in \operatorname{sp}(J)$, there exists a number $x_{0}$ in the closure $\Delta^{*}$ of $\Delta$ and a pair $y_{1}^{\prime}, y_{2}^{\prime}$ for which

$$
y_{1}^{\prime} \leqq y_{0} \leqq y_{2}^{\prime} ; x_{0}+i y_{1}^{\prime} \text { and } x_{0}+i y_{2}^{\prime} \text { in } \operatorname{sp}(T) .
$$

This implies

$$
\mu_{1}\left(\operatorname{sp}\left(J_{\Delta}\right)\right) \leqq I\left(\Delta^{*}\right),
$$

where $I(\delta)$ denotes the interval function defined by $I(\delta)=0$ if $\delta \cap \operatorname{Re}(\operatorname{sp}(T))$ is empty and $I(\delta)=\sup \operatorname{Im}(z)-\inf \operatorname{Im}(z)$ where $z \in \operatorname{sp}(T)$ and $\operatorname{Re}(z) \in \delta$.

Relation (9.5) now yields

$$
(2 \pi)^{1 / 2}\left\|C^{1 / 2} E(\Delta) f\right\| \leqq\left[d I\left(\Delta^{*}\right)\right]^{1 / 2}\|E(\Delta) f\| .
$$

If $(c, d]$ contains $\operatorname{sp}(H)$ and if $P: c=c_{0}<c_{1}<\cdots<c_{N}=d$ is a partition of $(c, d]$ into subintervals $\Delta_{k}=\left(c_{k-1}, c_{k}\right]$ then $I=\sum_{k=1}^{N} E\left(\Delta_{k}\right)$ and $\left\|C^{1 / 2} f\right\|$ $=\left\|C^{1 / 2} \sum_{k=1}^{N} E\left(\Delta_{k}\right) f\right\| \leqq \sum_{k=1}^{N}\left\|C^{1 / 2} E\left(\Delta_{k}\right) f\right\|$. An application of the Schwarz inequality to (9.17) then implies by virtue of $\|f\|^{2}=\sum_{k=1}^{N}\left\|E\left(\Delta_{k}\right) f\right\|^{2}$,

$$
(2 \pi)^{1 / 2}\left\|C^{1 / 2} f\right\| \leqq \sum_{k=1}^{N} d_{k} I\left(\Delta_{k}^{*}\right)^{1 / 2}\|f\|,
$$

where $d_{k}$ is the length of $\Delta_{k}$. If $F(x)$ is the function defined on $(c, d]$ by $F(x)=I\left(\Delta_{k}^{*}\right)$ on $\Delta_{k}$, then (9.18) becomes

$$
(2 \pi)^{1 / 2}\left\|C^{1 / 2} f\right\| \leqq\left(\int_{c}^{d} F(x) d x\right)^{1 / 2}\|f\| .
$$

Next, choose a sequence of partitions $\left\{P_{n}\right\}$ with the property that $P_{n+1}$ is a refinement of $P_{n}$ and such that the lengths of the intervals of $P_{n}$ tend to zero as $n \rightarrow \infty$. Let $F_{n}(x)$ correspond to $P_{n}$ as $F(x)$ does to $P$. It is clear from the definition of $M(x)\left(=M_{0}(x)\right)$ in $\S 3$ and the fact that $\operatorname{sp}(T)$ is a closed set that $I\left(\Delta^{n}\right) \rightarrow M(x)$ as $n \rightarrow \infty$, whenever $\left\{\Delta^{n}\right\}$ is any sequence of intervals containing $x$ for which $\Delta^{n} \rightarrow x$ as $n \rightarrow \infty$. Consequently, $F_{n}(x) \rightarrow M(x)$ as $n \rightarrow \infty$ for all $x$ on $(c, d]$, except 
possibly for those numbers $x$ in the (denumerable) set of partitioning points. Since $0 \leqq F_{n}(x) \leqq$ const., it then follows from (9.19) and from Lebesgue's term by term integration theorem that (3.2) holds with $\theta=0$. Since the same argument also applies to $T_{\theta}$, relation (3.2) is seen to hold for any real $\theta$.

Finally, by considering coverings of $S$ by denumerable unions of the type $\Sigma \Delta_{k}$, where $\Delta_{k}=\left(a_{k}, b_{k}\right]$ and the $\Delta_{k}$ are pairwise disjoint, a similar argument leads to

$$
(2 \pi)^{1 / 2}\left\|C^{1 / 2} E(S) f\right\| \leqq\left(\int_{S} M_{\theta}(x) d x\right)^{1 / 2}\|f\|,
$$

for any Borel set $S$ of the real line. Since, for any operator $A,\|A\|^{2}=\left\|A^{*} A\right\|$ and since $E(S) C E(S)=\left(C^{1 / 2} E(S)\right)^{*}\left(C^{1 / 2} E(S)\right)$, relations (9.20) and $D=2 C$ yield (3.4). This completes the proof of Theorem II.

10. Proof of (i) of Theorem III. For convenience it can be supposed that $\theta=0$. A similar argument with $T$ replaced by $T_{\theta}$ takes care of the general case. It follows from (3.4) that if $M_{\theta}(x)=0$ a.e. on $S$ then $C^{1 / 2} E(S)=0$, where $E(\lambda)$ is defined by (9.1), and hence

$$
C E(S)=0 \text {. }
$$

Multiplication of (1.3) on the left by $H$ leads to $H^{2} J-H J H=i H C$ while multiplication on the right leads to $H J H-J H^{2}=i C H$, hence

$$
H^{2} J-J H^{2}=i(H C+C H) .
$$

Since, by (10.1), $C f=0$ whenever $f \in \mathfrak{H}_{S}=E(S) \mathfrak{H}$ and since also $H f \in \mathfrak{H}_{s}$, then (10.2) implies that $\left(H^{2} J-J H^{2}\right) f=0$ for $f \in \mathfrak{H}_{s}$. Similarly $\left(H^{n} J-J H^{n}\right) f=0$ and hence $p(H) J f=J p(H) f$ for $f \in \mathfrak{H}_{S}$ and for any polynomial $p(H)$. On choosing a sequence of polynomials $p_{u}(H)$ converging (strongly) to $E(S)$, one obtains

$$
E(S) J f=J E(S) f .
$$

Consequently, $\mathfrak{H}_{S}$ is invariant under $J$ (as well as under $H$ ). Thus $T$ is reduced by $\mathfrak{H}_{S}$ and, by (10.1), $T$ is normal on $\mathfrak{H}_{s}$. Consequently, from the assumption $\mathfrak{S}=\Omega$ and the definition (4.1) of $\Omega, \mathfrak{H}_{S}=0$, that is, $E(S)=0$. This completes the proof of (i) of Theorem III.

11. Proof of (ii) of Theorem III. As above, it can be supposed that $\theta=0$. Let $T^{\prime}$ have the Cartesian representation

$$
T^{\prime}=H^{\prime}+i J^{\prime} \quad\left(H^{\prime}=\int \lambda d E^{\prime}(\lambda)\right)
$$

on the Hilbert space $\mathfrak{H}^{\prime}=P \mathfrak{G}$. Since $M_{0}^{\prime}(x)=0$ a.e. on $S$, then, as above, $T^{\prime}$ is reduced by, and is normal on, $E^{\prime}(S) \mathfrak{S}^{\prime}=\mathfrak{H}_{s}^{\prime}$. Consequently, $T$ leaves invariant $\mathfrak{H}_{S}^{\prime}$ (and $T^{\prime}=T / P \mathfrak{H}$ is normal on $\mathfrak{H}_{S}^{\prime}$ ). If $D \leqq 0$, then $T$ is hyponormal on $\mathfrak{S}$ and 
consequently $T$ is reduced by $\mathfrak{H}_{s}^{\prime}$; Berberian $[1$, p. 161, problem 9]. In case $D \geqq 0$, then $T^{*}$ is hyponormal and the preceding argument implies that $T^{*}$ (hence also $T$ ) is reduced by $\mathfrak{H}_{S}^{\prime}$. In any case, $T$ is reduced by, and is normal on $\mathfrak{H}_{s}^{\prime}$. As before, this implies $\mathfrak{H}_{S}^{\prime}=0$, that is, $E^{\prime}(S)=0$.

12. Proof of Theorem IV. The proof begins with the relations (9.5)-(9.7). Suppose that neither endpoint of $\Delta=(a, b]$ belongs to $\operatorname{sp}(H)$. Then it will be shown that (9.7) can be replaced by the stronger relation

$$
\left(J-y_{0} I\right) f_{n} \rightarrow 0 \text {. }
$$

Since $x_{0} \in \operatorname{sp}\left(H_{\Delta}\right)$ then $x_{0}$ belongs to the closure of $\Delta$, and since the endpoints of $\Delta$ do not belong to $\operatorname{sp}(H)$, it is clear that $x_{0}$ is an interior point of $\Delta$. In addition, it follows from (9.6) (cf. (7.4)) that for $g_{n}=\left(J-y_{0} I\right) f_{n}$,

$$
\left\|\left(H-x_{0} I\right) g_{n}\right\|^{2}=\int\left(\lambda-x_{0}\right)^{2} d\left\|E(\lambda) g_{n}\right\|^{2} \rightarrow 0 .
$$

Since $x_{0}$ is interior to $\Delta$, relations (12.2) and (9.7) imply that $g_{n} \rightarrow 0$, that is, (12.1). (That $x_{0}$ be an interior point of $\Delta$ is crucial here.) It then follows from (9.6) and (12.1) that $z_{0}=x_{0}+i y_{0} \in \operatorname{sp}(T)$.

Thus, whenever $y_{0} \in \operatorname{sp}\left(J_{\Delta}\right)$ and the endpoints of $\Delta$ do not belong to $\operatorname{sp}(H)$, there exist some $z_{0} \in \operatorname{sp}(T)$ with $\operatorname{Im}\left(z_{0}\right)=y_{0}$ and $\operatorname{Re}\left(z_{0}\right)=x_{0} \in \Delta$. If now the $\Delta$-strip: $\{x \in \Delta, y$ arbitrary $\}$ is subdivided into a finite or an infinite number of rectangles by horizontal segments, then it is clear that $d \mu_{1}\left(\operatorname{sp}\left(J_{\Delta}\right)\right)$ is not greater than any sum $S_{\Delta}$ of the areas of those rectangles containing points of $\operatorname{sp}(T)$. Hence, by $(9.5)$,

$$
(2 \pi)^{1 / 2}\left\|C^{1 / 2} E(\Delta) f\right\| \leqq S_{\Delta}^{1 / 2}\|E(\Delta) f\| .
$$

Now, in order to prove Theorem IV, suppose that (5.1) fails to hold, so that either $\operatorname{sp}(H)$ or $\operatorname{sp}(J)$ fails to contain an interval. There is no loss of generality in supposing that $\operatorname{sp}(H)$ does not contain an interval. Let $(\alpha, \beta]$ contain $\operatorname{sp}(H)$, hence, by Theorem $\mathrm{I},(\alpha, \beta]$ contains $\operatorname{Re}(\operatorname{sp}(T))$. Suppose also that $\alpha$ and $\beta$ do not belong to $\operatorname{sp}(H)$, and consider subdivisions of $(\alpha, \beta]:(\alpha, \beta]=\Sigma \Delta_{k}$, consisting either of a finite or of an infinite (denumerable) union of disjoint subintervals $\Delta_{k}$ of the type $\Delta$ and for which no endpoints of the $\Delta_{k}$ lie in $\operatorname{sp}(H)$. Then, by (12.3), $(2 \pi)^{1 / 2}\left\|C^{1 / 2} E\left(\Delta_{k}\right) f\right\| \leqq S_{\Delta k}{ }^{1 / 2}\left\|E\left(\Delta_{k}\right) f\right\|$ and therefore, by the Schwarz inequality,

$$
(2 \pi)^{1 / 2}\left\|C^{1 / 2} f\right\| \leqq\left(\Sigma S_{\Delta_{k}}\right)^{1 / 2}\|f\|
$$

Hence,

$$
2 \pi\|C\| \leqq \Sigma S_{\Delta_{k}} .
$$

Since $\operatorname{sp}(H)$ does not contain an interval it is clear that $\mu_{2}(\operatorname{sp}(T))=\inf _{P, S_{\Delta_{k}}} \sum S_{\Delta_{k}}$, 
where only those partitions $P$ (finite or infinite) are allowed with points not belonging to $\mathrm{sp}(H)$. The desired relation (3.6) now follows from (12.5).

13. Proof of Theorem V. It is sufficient to prove the theorem for $H$. It will be shown that the set consisting of the union of all open intervals contained in $\mathrm{sp}(H)$ is dense in $\mathrm{sp}(H)$. If the assertion were false, there would exist some closed interval $K$ containing a point of $\operatorname{sp}(H)$ in its interior, in particular,

$$
E(K) \neq 0,
$$

with the property that no subinterval of $K$ belongs to $\mathrm{sp}(H)$.

It follows from the argument of $\$ 12$ however that

$$
(2 \pi)^{1 / 2}\left\|C^{1 / 2} E(K) f\right\| \leqq\left[\mu_{2}\{z: z \in \operatorname{sp}(T) \text { and } \operatorname{Re}(z) \in K\}\right]^{1 / 2}\|E(K) f\| .
$$

Since (5.4) implies that the right side of (13.2) is 0 , it follows that $C^{1 / 2} E(K)=0$, hence

$$
C E(K)=0 .
$$

As in $\$ 10$ (cf. (10.1)), it follows that $T$ is reduced by, and is normal on, $E(K) \mathfrak{H}$. Since $\mathfrak{D}=\Omega$, then $E(K)=0$, in contradiction with (13.1), and the proof of Theorem $\mathrm{V}$ is complete.

14. Toeplitz matrices. Let $\left\{c_{n}\right\}$ for $n=1,2, \cdots$, be a sequence of complex numbers for which the power series

$$
f(z)=\sum_{n=1}^{\infty} c_{n} z^{n}
$$

is bounded on the open disk $|z|<1$. Let $T=\left(t_{j k}\right)$, for $j, k=1,2, \cdots$, be defined by

$$
t_{j k}=c_{k-j} \text { if } k-j \geqq 1 \text { and } t_{j k}=0 \text { otherwise. }
$$

Then $T$ is known to be bounded and its spectrum is the closure of the set of values $f(z)$ when $|z|<1$; Wintner [13, p. 279]. Furthermore, $T$ is semi-normal; in fact

$$
T T^{*}-T^{*} T=D, \text { where } D=B^{*} B \text { and } B=\left(c_{j+k-1}\right) ;
$$

$[7$, p. 517], [8, p. 838].

It will be supposed that $T$ is not normal, so that not all $c_{k}$ are zero. Then $\operatorname{sp}(T)$ is the closure of a connected open set and hence its projections on the real and imaginary axes are closed intervals. According to Theorem I, the spectra of the real and imaginary parts of $T$ are then closed intervals. This last result was first proved by Hartman and Wintner [3, p. 868].

According to Corollary 2 of Theorem $I, W(T)$ is the closed convex hull of $\operatorname{sp}(T)$. This result is also known; see Wintner [13, p. 278].

It is noteworthy that all examples of non-normal, semi-normal operators 
furnished by (14.2) do have spectra with positive two dimensional Lebesgue measures. Whether also relation (3.6) holds for these operators, in general (that is with ut any restriction on $\operatorname{sp}(T)$ such as, for instance, that made in Corollary 2 of Theorem II) will remain open, although (3.5) and even (3.2) always hold.

If $T$ is defined by $(14.2)$ then $\mu_{2}(\operatorname{sp}(T))$ is not greater than the double integral of $\left|f^{\prime}(z)\right|^{2}$ taken over the disk $|z| \leqq 1$, hence,

$$
\mu_{2}(\operatorname{sp}(T)) \leqq \pi \sum_{k=1}^{\infty} k\left|c_{k}\right|^{2}
$$

with the equality surely holding if the mapping $z \rightarrow f(z)(|z|<1)$ is one to one. It follows from (14.3) however (cf. [7, p. 517]) that for $x=\left(x_{1}, x_{2}, \cdots\right) \in \mathfrak{S}$, $(D x, x)=\sum_{j=1}^{\infty}\left|\sum_{k=1}^{\infty} c_{j+k-1} x_{k}\right|^{2}$, and hence, using the Schwarz inequality,

$$
\|D\| \leqq \sum_{k=1}^{\infty} k\left|c_{k}\right|^{2}
$$

Consequently, relation (3.6) certainly holds whenever equality holds in (14.4).

15. Singular integral operators. Another class of semi-normal operators is given by the operators $T=H+i J$, where

$$
H f=h(x) f(x)+(1 / i \pi) \phi(x) \int_{a}^{b} \phi^{*}(t)(t-x)^{-1} f(t) d t
$$

and

$$
J f=x f(x),
$$

where $f \in \mathfrak{H}=L^{2}(a, b),-\infty<a<b<\infty$, and $h(x)$ is real-valued, $\phi(x)$ is complexvalued, and both $h$ and $\phi$ are bounded and measurable on $(a, b)$. See [9] and the references cited there.

If $D$ is defined by (1.1) and (1.3), then $\|D\|=2 \pi^{-1}\|\phi\|^{2}$ (cf. [9]) and (3.5) becomes

$$
2 \int_{a}^{b}|\phi(t)|^{2} d t \leqq \mu_{2}(W(T))
$$

in case $\phi(x) \equiv 1$, this becomes

$$
2(b-a) \leqq \mu_{2}(W(T)) .
$$

In case also $h(x) \equiv 0$, that is, if $T_{0}=H_{0}+i J$ is defined by (15.2) and

$$
H_{0} f=(1 / i \pi) \int_{a}^{b}(t-x)^{-1} f(t) d t,
$$

then $\operatorname{sp}\left(H_{0}\right)=[-1,1]$ (Koppelman and Pincus [4], see also [9]) while $\operatorname{sp}(J)=[a, b]$. Hence, if $R$ denotes the closed rectangle $R=\{(x, y):-1 \leqq x \leqq 1, a \leqq x \leqq b\}$, then, by (15.4), $R=W\left(T_{0}\right)$. 
Addendum (May 25, 1965). The assertions of the two corollaries of Theorem I have also been proved by Stampfli (Hyponormal operators and spectral density, Trans. Amer. Math. Soc. 117 (1965), 469-476).

Concerning the sets $\operatorname{sp}(T)$ and $W(T)$, see the remarks on p. 482 of Berberian (The numerical range of a numerical operator, Duke Math. J. 31 (1964), 479-484) and the reference given there to Schreiber; also, as was called to the author's attention by the referee, the paper of Orland (On a class of operators, Proc. Amer. Math. Soc. 15 1964), 75-79).

Relative to some of the results of $\S 4$, it can be noted that in the above mentioned paper of Stampfli, he has proved that if $T$ is semi-normal and if $\operatorname{sp}(T)$ is a subset of a smooth simple closed curve, then $T$ is normal. Thus the possibility suggested at the end of $\S 4$ that an isolated part of $\operatorname{sp}(T)$ (when $\mathfrak{H}=\Omega$ ) might be such a curve can now be ruled out.

\section{REFERENCES}

1. S. K. Berberian, Introduction to Hilbert space, Oxford Univ. Press, New York, 1961.

2. P. R. Halmos, Introduction to Hilbert space, Chelsea, New York, 1951.

3. P. Hartman and A. Wintner, The spectra of Toeplitz's matrices, Amer. J. Math. 76 (1954), 867-882.

4. W. Koppelman and J. Pincus, Spectral representations for finite Hilbert transformations, Math. Z. 71 (1959), 399-407.

5. C. R. Putnam, On commutators and Jacobi matrices, Proc. Amer. Math. Soc. 7 (1956), 1026-1030.

6. - On semi-normal operators, Pacific J. Math. 7 (1957), 1649-1652.

7. - Commutators and absolutely continuous operators, Trans. Amer. Math. Soc. 87 (1958), 513-525.

8. - On Toeplitz matrices, absolute continuity, and unitary equivalence, Pacific J. Math. 9 (1959), 837-846.

9. - Commutators, absolutely continuous spectra, and singular integral operators, Amer. J. Math. 86 (1964), 310-316.

10. F. Riesz and B. S7-Nagy, Functional analysis, Ungar, New York, 1955.

11. J. G. Stampfli, Hyponormal operators, Pacific J. Math. 12 (1962), 1453-1458.

12. M. H. Stone, Linear transformations in Hilbert space and their applications to analysis, Amer. Math. Soc. Colloq. Publ. Vol. 15, Amer. Math. Soc., Providence, R. I., 1932.

13. A. Wintner, Zur Theorie der beschränkten Bilinearformen, Math. Z. 30 (1929), 228-282.

PURdue UnIVERSITY,

LAFAYETTE, INDIANA 